\title{
Statistical properties of agent-based market area model
}

\author{
Zoltán Kuscsik, Denis Horváth \\ Department of Theoretical Physics and Astrophysics, University of \\ P.J. Šafárik, Košice, Slovak Republic
}

\begin{abstract}
One dimensional stylized model taking into account spatial activity of firms with uniformly distributed customers is proposed. The spatial selling area of each firm is defined by a short interval cut out from selling space (large interval). In this representation, the firm size is directly associated with the size of its selling interval.

The recursive synchronous dynamics of economic evolution is discussed where the growth rate is proportional to the firm size incremented by the term including the overlap of the selling area with areas of competing firms. Other words, the overlap of selling areas inherently generate a negative feedback originated from the pattern of demand. Numerical simulations focused on the obtaining of the firm size distributions uncovered that the range of free parameters where the Pareto's law holds corresponds to the range for which the pair correlation between the nearest neighbor firms attains its minimum.
\end{abstract}

\section{Introduction}

The study of elemental interactions in social and economical systems has a great importance to understand the large-scale system properties. One of the universal large-scale properties exhibited by social systems in a robust way is the Pareto's law of wealth distribution and firm size [3, 6, 10]. Pareto's law is generally associated to the observation, that personal income of individuals, the size of companies are distributed by power-law.

The formation of power-laws has generally complex origin. Among other 
approaches, highly sophisticated multi-agent models have been developed 2, 14, 15 to explain the power-laws observed in various social systems.

In this paper we propose agent-based model that emphasizes role of spatial location of firm within the limited market area. The model approximates the basic mechanisms of competency that simply follows from the spatial positions and selling activities of firms.

An extensive economic literature exists that deals with the competitiveness as consequence of location. One dimensional model of spatial duopoly introduced by Hotelling [ 8 , has assumed that consumers are continuously and uniformly distributed along a line segment. The model of firm distribution in a non-uniform environment has been developed by Lawrence [16. It predicts firm density in an urban setting in which the population density decreases exponentially with the distance from the center. Erlenkotter [5] has considered uniformly distributed demand over the infinite plane. He has discussed various regular two-dimensional market shapes. An elegant and advanced multistore competitive model of two firms in a finite business area has been introduced by Dasci and Laporte 4. This model has been investigated for one and two dimensional geographical markets. It assumes the costumers are dispersed through space in only one direction along some coordinate $x \in(0,1)$. In this regard it is useful to mention the functional expression for total revenue per firm

$$
\int_{0}^{1} Q(x) f(x) \mathrm{dx},
$$

where $f(x)$ is the probability density function of the customers multiplied by the probability $Q(x)$ that customer at $x$ patronizes product of given firm.

As we have mentioned before, our present approach also pays attention to spatial aspects. The approach comes from ecologic-economic feedback concept of regulated factory emissions introduced by us recently [9]. The work points out an emergence of critical properties in a two dimensional system with spatially distributed agents balancing the conflicting objectives. The model assumes that sources of diffusive emissions compete with the distributed sensorial agents. The analysis of the complex numerical data yield us to reductionist and purely geometric formulation that is related to coverage percolation problem. The geometric idea has been applied here to study the spatial distribution of the competitive firms reduced to basic geometric objects that cover market area. Our stylized spatial model is defined as it follows.

\section{Firm growth}

We assume that each firm acts as a seller agent of a product from the same sort of industry or it behaves as a provider of some service business. The spatial economic activity of the $i$ th agent is defined by its position $x_{i}^{(t)} \in(0, L)$ and by its selling area $\left(x_{i}^{(t)}-r_{i}^{(t)}, x_{i}^{(t)}+r_{i}^{(t)}\right) \bmod L$, where $L$ is the constant size of one dimensional market space with periodic boundary conditions and $r_{i}^{(t)}$ is the 
selling radius of firm $i$. It should be noted that radius does not mean strictly the space of the physical activity of the seller but it can be understood as a radius up to which the customers are attracted. We have considered customers uniformly distributed along a straight line. Interestingly, such arrangement is typical for the restaurants distributed along a main road or highway [4].

We follow with definition of the measure of the spatial activity of the $i$ th firm

$$
s_{i}^{(t)}(x)= \begin{cases}1 & \text { if } i \text { claims to sell at the position } x \\ 0 & \text { if } x \text { is not from the agent's selling area }\end{cases}
$$

Generally, large selling area means more potential consumers covered by delivery of given product which results higher interest connected to higher profit. Without negative economic feedback, the continuous investment of the constant fraction of income yields to the exponential growth of the firm size and its sale. Several facts that yield the negative feedback between firm and its environment should be mentioned: (i) the transportation costs of products are convex functions of distance [11]; (ii) the complexity of firm management grows with a firm size (iii) larger firms use more sophisticated and thus more expensive information technologies; (iv) the presence of two or more competitive products in the same location affects the prices as well as the annual sales.

Here we assume only a negative feedback that originates from the spatial overlap of selling areas. The overlap of $i$ th firm area with the areas of the remaining $(N-1)$ firms is defined by

$$
\Omega_{i}^{(t)}=\sum_{\substack{j=0 \\ j \neq i}}^{N} \int_{0}^{L} s_{i}^{(t)}(x) s_{j}^{(t)}(x) \mathrm{d} x
$$

With this firm-firm interaction picture in mind, we suggested the dynamical rule of the firm growth

$$
r_{i}^{(t+1)}=r_{i}^{(t)}+\alpha r_{i}^{(t)}-\beta \Omega_{i}^{(t)},
$$

where $\alpha>0$ and $\beta>0$ are constant parameters controlling the instantaneous growth. The term $-\beta \Omega_{i}^{(t)}$ can be interpreted as a negative feedback that reflects the competition. The selling area of firm $i$ is expressed by

$$
S_{i}^{(t)}=\int_{0}^{L} s_{i}^{(t)}(x) \mathrm{d} x=2 r_{i}^{(t)} .
$$

\section{$3 \quad$ Firm establishment and bankruptcy}

In the stylized version of the model we study the firm is established at random position with a small random initial selling radius $r_{i}^{(t)} \in\left(r_{\mathrm{a}}, r_{\mathrm{b}}\right)$, where $r_{\mathrm{a}}$ is assumed to be the lower bound of profitability (smallest firm). Therefore, the 
bankruptcy of a firm occurs when $r_{i}^{(t)}<r_{\mathrm{a}}$. At the same time new firm (with the same index) is established at a new random position with some initially random size. This death-birth process is analogous to the so called extremal dynamics principle [1] applied to e.g. models of the wealth distribution [12] and stock markets [7].

\section{Simulation and Numerical results}

The choice of parameters is chronic problem specially in models of the socialeconomic systems. Although models of interacting agents give qualitative predictions that in many aspects resemble behavior of real-world systems, in the most cases, the quantitative analysis needs laborious tuning of parameters until the range is reached for which the phenomenon of interest takes place. Our simulations were performed with constant number of firms $N=500$ for predefined market area $L=3 \times 10^{5}$. The constant growth factor $\alpha=0.01$ and the initial range of the selling space constrained by radii $r_{\mathrm{a}}=2.0$ and $r_{\mathrm{b}}=5.0$ is chosen to invoke steps much smaller than $L$.

To reach the stationary regime $10^{5}$ the initial synchronous system updates were discarded. The information from subsequent $10^{6}$ updates spent in stationary regime has been recorded. Their analysis has uncovered that firm-firm interaction controlled by $\beta$ admits to establish market regimes that differ in size distributions. We observed that sufficiently large $\beta$ leads to the market with lowered overlaps. On other hand, sufficiently small $\beta$ supports formation of oligopoly that cover a dominant area of available market space. The important for us power-law distributions are observable only for exceptional $\beta$ (see fig (4). This finding opens a question: what regulatory real-world economic principle controls the sustaining of the empirically relevant power-law regimes. The related question is the optimization of free parameters. For this purpose we examined several heuristic criteria. The most attractive seems to be an extremal entropy principle [13], but in that case one faces to the usual problem of the proper entropy definition.

More pragmatic, however, less fundamental attempt comes from our analysis of firm-firm correlations. For this purpose the pair correlation function $C$ of sizes of nearest neighboring firms $(k)$ at positions $x_{i}^{(t)} \leq x_{k}^{(t)}$ can be defined as

$$
C=\left\langle\frac{\frac{1}{N} \sum_{i=1}^{N} r_{i}^{(t)} r_{k}^{(t)}-\left(\frac{1}{N} \sum_{i=1}^{N} r_{i}^{(t)}\right)^{2}}{\frac{1}{N} \sum_{i=1}^{N}\left(r_{i}^{(t)}\right)^{2}-\left(\frac{1}{N} \sum_{i=1}^{N} r_{i}^{(t)}\right)^{2}}\right\rangle_{t}
$$

Here $\langle\ldots\rangle_{t}$ denotes temporal stationary average. The calculations for different $\beta$ uncovered that minimum of correlation function corresponds to parameter (or narrow interval of parameters) for which nearly power-law distributions can be fitted quite well. This extremal principle reflects the possible importance of the measurements of the spatial correlations in social and economic systems. 


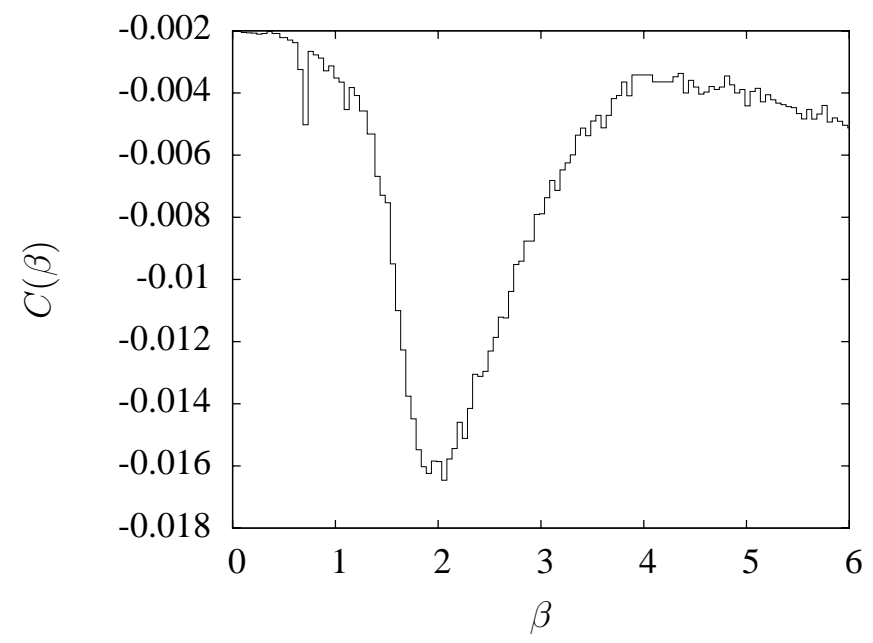

Figure 1: Plot of the pair correlation function defined by Eq. 6 as a function of $\beta$. The Pareto's law for the firm size belongs to $\beta$ where $C(\beta)$ attains its minimum.

a.)
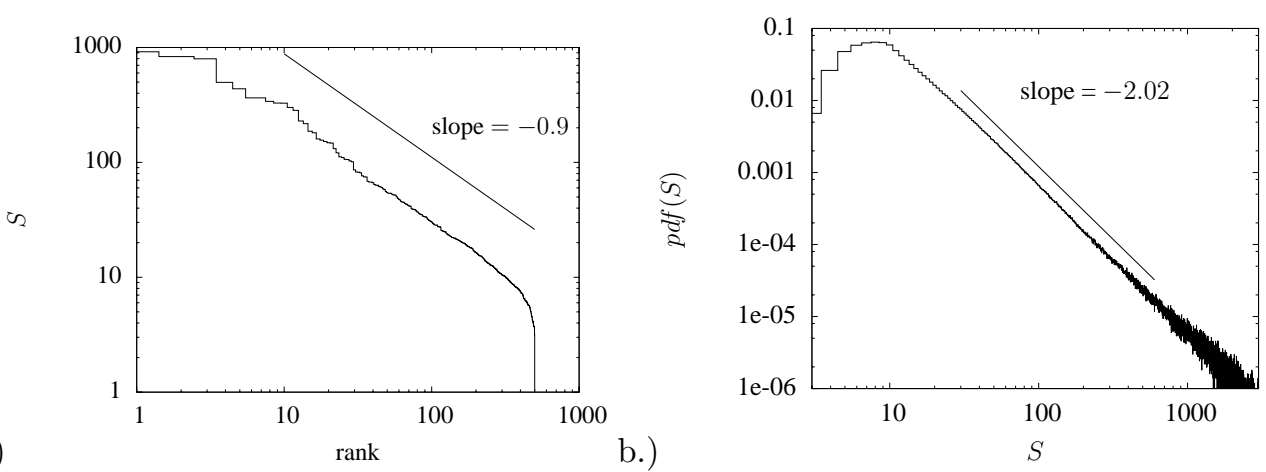

Figure 2: a.) Plot of sizes of firms as a function their rank for $\beta=2.0$. The fitted power-law index is close to unity. b.) The distribution of firm sizes.

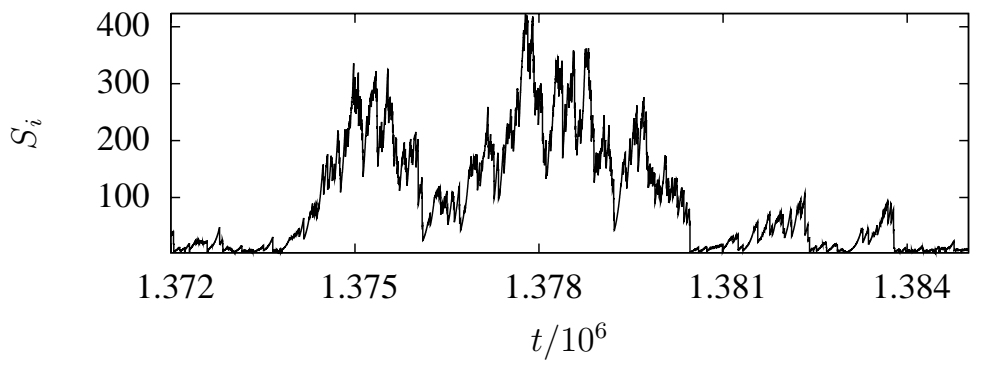

Figure 3: The time evolution of size of selected firm. 


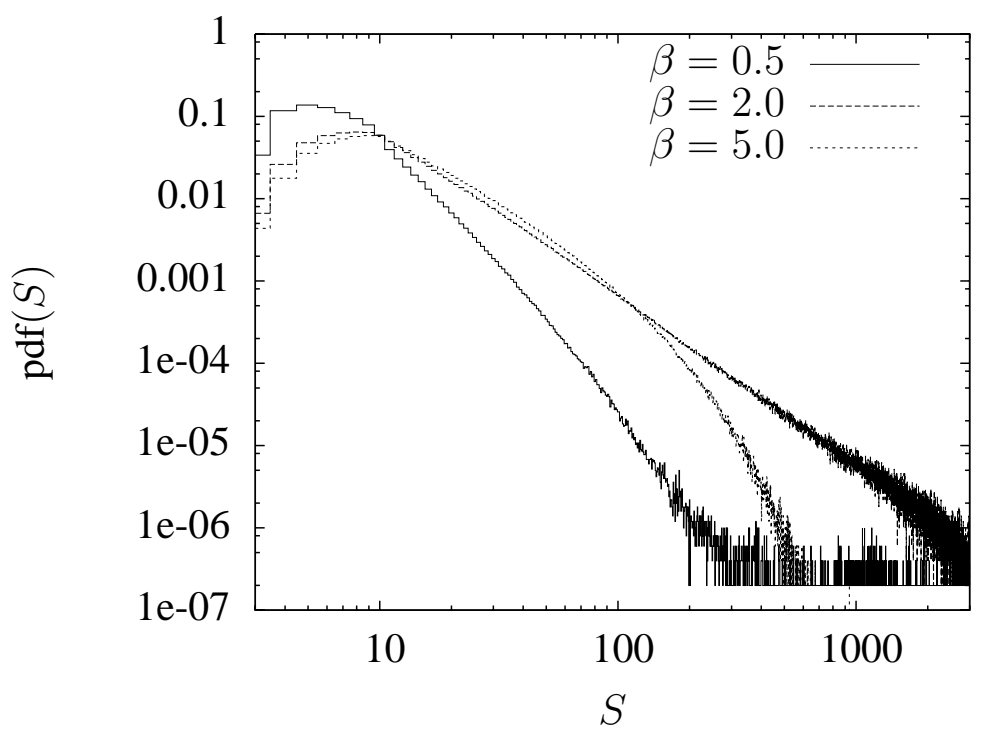

Figure 4: Distribution of firm sizes plotted for different $\beta$.

\section{Conclusions}

By focusing on the geometric representation of firms we proposed a stylized multi-agent model of firm growth. The competitive dynamics of firms under which the system reaches a steady state results a complex patterns of firm locations. Despite of its formal simplicity, the model supplemented by the principle of minimum firm-firm correlation is able to explain the origin of the Pareto's law. Further validating of model is planned that takes into account real-world data. Hoverer, this will need to take into account the non-uniform distribution of customers. The advanced model of this type is under development.

Acknowledgments The authors would like to express their thanks to Slovak Grant agency LPP APVV (grant no. 0098-06), VEGA (grant no. 1/4021/07, $1 / 2009 / 05)$ for financial support.

\section{Bibliography}

[1] Boettcher, Stefan, and Allon G. Percus, "Optimization with extremal dynamics", Phys. Rev. Lett. 86, 23 (Jun 2001), 5211-5214.

[2] Bouchaud, Jean-Philippe, and Marc Mezard, "Wealth condensation in a simple model of economy", Physica A 282 (Jul 2000), 536-545.

[3] Clementi, F., T. Di Matteo, and M. Gallegati, "The power-law tail exponent of income distributions", Physica A 370, 1 (Oct 2006), 49-53. 
[4] Dasci, Abdullah, and Gilbert Laporte, "A continuous model for multistore competitive location", Oper. Res. 53, 2 (2005), 263-280.

[5] Erlenkotter, Donald, "The general optimal market area model", Annals of Operations Research 18, 1 (Dec 1989), 43-70, 10.1007/BF02097795.

[6] Hegri, Geza, Zoltan Neda, and Maria Augusta Santos, "Wealth distribution and pareto's law in the hungarian medieval society", Physica A 380 (Jul 2007), 271-277.

[7] Horvath, D., and Z. Kuscsik, "Structurally dynamic spin market networks", ArXiv - physics/0701156 (2007).

[8] Hotelling, Harold, "Stability in competition", The Economic Journal 39, 153 (Mar 1929), 41-57.

[9] Kuscsik, Zoltan, Denis Horvath, and Martin Gmitra, "The critical properties of the agent-based model with environmental-economic interactions", Physica A 379, 1 (Jun 2007), 199-206.

[10] Malevergne, Yannick, and Didier Sornette, "A two-factor asset pricing model and the fat tail distribution of firm sizes", SSRN eLibrary (2007).

[11] MCCann, Philip, "Transport costs and new economic geography", J Econ Geogr 5, 3 (2005), 305-318.

[12] Pianegonda, S., J. R. Iglesias, G. Abramson, and J. L. Vega, "Wealth redistribution with conservative exchanges", Physica A: Statistical Mechanics and its Applications 322 (May 2003), 667-675.

[13] Raine, Alan, John Foster, and Jason Potts, "The new entropy law and the economic process", Ecological Complexity 3, 4 (Dec 2006), 354-360.

[14] Rawlings, Philip K., David Reguera, and Howard Reiss, "Entropic basis of the pareto law", Physica A $\mathbf{3 4 3}$ (Nov 2004), 643-652.

[15] Samanidou, E, E Zschischang, D Stauffer, and T Lux, "Agent-based models of financial markets", Reports on Progress in Physics 70, 3 (2007), 409-450.

[16] White, Lawrence J., "The spatial distribution of retail firms in an urban setting", Regional Science and Urban Economics 5, 3 (August 1975), 325333. 